\title{
Two-Dimensional CFD Modeling of Wave Rotor Flow Dynamics
}

Gerard E. Welch and Rodrick V. Chima

Lewis Research Center

Cleveland, Ohio

Prepared for the

11th Computational Fluid Dynamics Conference

sponsored by the American Institute of Aeronautics and Astronautics Orlando, Florida, July 6-9, 1993

\section{N/SA}




\title{
Two-Dimensional CFD Modeling of Wave Rotor Flow Dynamics
}

\author{
Gerard E. Welch* and Rodrick V. Chima** \\ NASA Lewis Research Center \\ Cleveland, $\mathrm{OH} 44135$
}

\begin{abstract}
$\underline{\text { Abstract }}$
A two-dimensional Navier-Stokes solver developed for detailed study of wave rotor flow dynamics is described. The CFD model is helping characterize important loss mechanisms within the wave rotor. The wave rotor stationary ports and the moving rotor passages are resolved on multiple computational grid blocks. The finite-volume form of the thin-layer Navier-Stokes equations with laminar viscosity are integrated in time using a four-stage Runge-Kutta scheme. Roe's approximate Riemann solution scheme or the computationally less expensive Advection Upstream Splitting Method (AUSM) flux-splitting scheme is used to effect upwind-differencing of the inviscid flux terms, using cell interface primitive variables set by MUSCLtype interpolation. The diffusion terms are centraldifferenced. The solver is validated using a steady shock/laminar boundary layer interaction problem and an unsteady, inviscid wave rotor passage gradual opening problem. A model inlet port/passage charging problem is simulated and key features of the unsteady wave rotor flow field are identified. Lastly, the medium pressure inlet port and high pressure outlet port portion of the NASA Lewis Research Center experimental divider cycle is simulated and computed results are compared with experimental measurements. The model accurately predicts the wave timing within the rotor passage and the distribution of flow variables in the stationary inlet port region.
\end{abstract}

\section{Introduction}

The wave rotor is an internal flow device used to transfer energy between gas streams of differing energy density. The energy exchange is accomplished through a timed sequence of unsteady compression and expansion wave processes that take place within the wave rotor passages which are arranged circumferentially at the periphery of the cylindrical rotor as shown in Figure 1. The waves are initiated as the ends of the wave rotor

\footnotetext{
*National Research Council Research Associate Member AIAA

**Aerospace Engineer, Member AIAA
}

passages sequentially open (or close) to stationary inlet and outlet ports. At an inlet port the low pressure gas in a rotor passage is exposed to the high pressure "driver" gas and a compression wave moves into the passage, compressing the "driven" gas on-board the rotor passage. The contact discontinuity between the driver gas and the driven gas follows the compression wave as the driver gas enters the passage and an expansion wave propagates into the inlet port region. At an outlet port, the typically higher pressure passage gas is exposed to the low pressure port flow and an expansion wave moves into the passage, reducing the on-board pressure, and exhausting the on-board gas to the outlet port. The charging and exhausting processes are used in the wave rotor to effect different gas cycles, including three-port divider cycles', like the NASA LeRC wave rotor experiment described below, in which a medium stagnation pressure stream is split into a high stagnation pressure stream and a low stagnation pressure stream, and four-port Brayton topping-cycles, with application in propulsion systems. ${ }^{2}$

The wave rotor presents a challenging application for high resolution Navier-Stokes schemes. The numerics must resolve and accurately track the shock waves and contact discontinuities and model strong vortical flows resulting from unsteady shock/boundary layer interaction and shock/contact interaction. A two-dimensional $(\theta, z)$ thin-layer Navier-Stokes solver has been developed for detailed study of the wave rotor flow dynamics. The multi-block solver uses high resolution upwinddifferencing of the inviscid flux terms using either the Roe approximate Riemann solution scheme ${ }^{3}$ or the AUSM flux splitting scheme $e^{4,5}$ and central-differencing of the diffusion terms.

The present modeling effort supports fundamental experimental ${ }^{6}$ and computational ${ }^{7,8}$ wave rotor research which is currently underway at the NASA Lewis Research Center aimed at characterizing the loss mechanisms due to friction, leakage, and gradual passage opening-time effects. A detailed study of the effects of geometry (e.g., inlet port lip rounding and passage profiling) on the shape and evolution of the contact discontinuity, on vortical flow structures generated within the rotating rotor passages that lead to mixing losses, and on the unsteadiness and nonuniformity of the flow in the stationary ports was conducted using the solver described here. ${ }^{9}$ 
A detailed overview of the CFD modeling of wave rotor flow dynamics in the $(\theta, z)$-dimensions is presented in this paper. A model wave rotor problem, specifically developed to study the inlet port/passage charging process is first described. This description identifies some of the important features of the wave rotor flow field. The numerical modeling is then provided, followed by examples of CFD and experimental model validation. Computed results for the model inlet port problem are presented. The results have helped identify the key features of the wave rotor flow field. Finally, computed results of a simulation of the medium and high pressure port portion of the NASA LeRC divider cycle are compared with experimental measurements.

\section{Model Inlet Port Problem Description}

Figure 2 provides a schematic diagram showing a group of rotor passages moving past an inlet port. In this view (looking radially-inward), the wave rotor passages are unwrapped from the periphery of the rotor. The right boundary of the rotor passages is a wall. (Note that the portion of the NASA LeRC divider cycle considered later in this paper is similar to the model problem shown here; however, in the divider cycle, a portion of the right-hand wall is replaced by the outlet port.)

During the charging process shown, a lower pressure "virgin" passage is exposed to a higher total pressure inlet port flow. As the virgin passage gradually opens to the higher total pressure inlet port flow, a compression wave, eventually steepening into a shock, forms at the leading end of the passage and moves into the rotor passage. This wave is followed by a contact discontinuity between the driver fluid and the driven fluid as shown in Figure 2. During the gradual opening, an expansion wave is generated and moves into the inlet port region, reducing the total pressure of the driver fluid as it enters the passage. The rightward running shock wave compresses the on-board (driven) gas and eventually reflects off the right-hand wall. The leftward running reflected shock recompresses the driven gas, interacts with the contact discontinuity, and compresses the driver gas within the passage. The inlet port is designed so that the rotating passages close just as the reflected shock reaches the left end of the rotor passage.

\section{Computational Model}

The unsteady quasi-3-D rotor viscous code (RVCQ3D) of Chima ${ }^{10}$ provided a skeletal structure for the finitevolume code (WROTOR2) developed in this work. Both single-passage and multi-passage/inlet port computations have been used to model the wave rotor flow field. In a single-passage calculation, the flow in a single rotor passage is modeled as that passage moves past either walls or inlet and outlet ports.
An example multi-block computational domain is shown in Figure 2 and includes the inlet port region and the multiple rotor passages that can influence flow in the inlet port. The inlet port is modeled with a stationary computational block while the moving rotor passages are each resolved on separate, though identical, computational blocks. The walls separating the individual passages are infinitely thin in the current work except at the leading edge where profiling the passage wall can be included. To date, any outlet ports (e.g., like those of the NASA LeRC divider cycle discussed below) influencing a simulation are simply represented by uniform static pressure surfaces rather than by separate computational blocks. Modifying the multi-block solver to include outlet port blocks is a straight-forward extension of the work presented here.

\section{Model Restrictions}

In general, centrifugal forces acting on the gas within the rotor passages work to establish nonuniform, radially stratified flow. The centrifugal effects provide a mechanism for distortion or instability (and mixing) at the contact discontinuity for driver and driven gases of differing density ${ }^{11}$; however, the degree to which this mechanism is important might be expected to scale with the square of rotor speed (or wheel Mach number) and the relative height of the rotor passage to the radius of the rotor. Influenced by the relatively low rotor speeds $(4000$ to $8000 \mathrm{rpm})$ and the dimensions $(\Delta \mathrm{r} / \mathrm{R} \simeq 0.067)$ of the NASA LeRC experiment, in this study both centrifugal and Coriolis forces are neglected by choosing to model the rotor passages and inlet ports in the $\theta-z$ (or $y-x)$ coordinates shown in Figure 2. Leakage flows, both from the rotor passages to the space between the rotor and the outer casing, and from passage to passage, are not yet included in this 2-D model. Fluid-to-wall heat transfer is another potential loss mechanism neglected in the calculations presented here as the blades separating the passages are treated as adiabatic surfaces.

\section{Governing Equations}

The unsteady, compressible 2-D thin-layer NavierStokes equations with laminar viscosity are used to model the wave rotor working fluid, which is treated as a calorically and thermally perfect gas. The governing equations in boundary-fitted coordinates $(\xi, \eta)$ under the thin-layer approximation ${ }^{12}$ are

$$
\frac{\partial Q}{\partial t}+\frac{\partial E}{\partial \xi}+\frac{\partial G}{\partial \eta}=\frac{\partial G_{x}}{\partial \eta}
$$

where $Q=(\rho, \rho \mathrm{u}, \rho \mathrm{v}, \rho e)^{\mathrm{T}} / \mathrm{J}$ is the vector of conserved variables, $\underline{F}$ and $\underline{G}$ are the inviscid flux vectors given by 


$$
E=\frac{|\nabla \xi|}{J}\left[\begin{array}{c}
\rho \tilde{U} \\
\rho u \tilde{U}+\xi_{x} p \\
\rho v \tilde{U}+\xi_{y} p \\
\rho h \tilde{U}-\xi_{t} p
\end{array}\right] \text { and } \varepsilon=\frac{|\nabla \eta|}{J}\left[\begin{array}{c}
\rho \tilde{V} \\
\rho u \tilde{V}+\tilde{\eta}_{x} p \\
\rho v \tilde{V}+\tilde{\eta}_{y} p \\
\rho h \tilde{V}-\tilde{\eta}_{t} p
\end{array}\right]
$$

and where $G_{\text {, is }}$ the viscous flux vector given by

$$
\underline{G_{y}}=\frac{|\nabla \eta|}{J}\left[\tilde{\eta}_{x}\left(\begin{array}{c}
0 \\
\tau_{x x} \\
\tau_{x y} \\
(\underline{I} \cdot)_{x}-q_{x}^{\prime \prime}
\end{array}\right)+\tilde{\eta}_{y}\left(\begin{array}{c}
0 \\
\tau_{x y} \\
\tau_{y y} \\
\left.(\underline{\underline{\tau}} \nu)_{y}-q_{y}^{\prime \prime}\right)
\end{array}\right)\right]
$$

$\tilde{U}=\nabla \xi \cdot(\underline{u}-\dot{x})$ and $\tilde{V}=\nabla \tilde{\eta} \cdot(\underline{u}-\dot{x})$ are the $\xi$ and $\eta$ normalized contravariant velocity components relative to the grid motion, $u$ and $v$ are the axial and tangential ( $x$ and $y)$ components of velocity, $\dot{z}$ is the grid velocity, $\rho$ is the fluid mass density, $p$ is the static pressure, $e$ is the total specific energy, $h$ is the total specific enthalpy, $(\underline{\underline{\tau}} u)_{x}=\tau_{x x x} u+\tau_{x y} V$, and $(\underline{\underline{\tau}} \underline{u})=\tau_{x y} u+\tau_{y y} V$. The shear stress (e.g., $\tau_{x y}$ ) and heat flux components (e.g., $\left.q_{y}^{\prime \prime}\right)$ are also expanded in the boundary-fitted coordinates and, under the thin-layer approximation, all derivatives with respect to the streamwise coordinate $(\xi)$ are neglected. The normalized metric terms (e.g., $\left.\xi_{x}=\xi_{x} /|\nabla \xi|\right)$ and the surface areas of the finitevolumes (e.g., $|\nabla \xi| / J$ where $J$ is the Jacobian of the $(x, y)-(\xi, \eta)$ transformation) are calculated using the specified grid. In this work, the grid speed metric $\xi_{t}$ is zero while $\eta_{t}=-\bar{\Gamma} \omega$ where $\bar{r}$ is the radius at which the calculations take place (e.g., the passage average radius) and $\omega$ is the angular velocity of the rotor.

\section{Time Integration}

The governing equations in finite-volume form are integrated in time using a second-order accurate explicit four-stage Runge-Kutta scheme (see Jameson et al. ${ }^{13}$ ). The time step is dictated in part by the CFL number, $\sigma$, which is nominally set to 1.2 . For solutions of steady flows or for unsteady inviscid flow fields resolved on nearly uniform grids (on which the CFL number is nearly uniform in space), the implicit residual smoothing ${ }^{14}$

$$
\left(1-\epsilon_{\xi} \delta_{\xi \xi}\right)\left(1-\epsilon_{\eta} \delta_{\eta \eta}\right) \bar{R}_{k}=R_{k}
$$

where $\delta_{\xi \xi}$ and $\delta_{\eta \eta}$ are central-difference second derivative operators, can be applied after each $\mathbf{k}^{\text {th }}$ RungeKutta step to smooth the residuals $R_{k}=(\partial Q / \partial t)_{k}$ so that the local CFL number can be increased. In this work the smoothing coefficient suggested by Turkel and van Leer $^{15}, \epsilon_{\xi}=0.9 \sigma / 8$ is used for the $\xi$-direction smoothing and $\epsilon_{\eta} \approx 0.5$ is used for the $\eta$-direction smoothing. Typically, $\sigma=3$ is used when the residuals are smoothed. The residual smoothing adds approximately a 3 to $5 \%$ overhead to the overall $\mathrm{cpu}$ time.

\section{Spatial Discretization}

In the cell-centered finite-volume formulation, the inviscid and viscous flux vectors are calculated at the cell interfaces which are constructed using lines connecting the grid points. The shear stress and heat flux components of the viscous flux vector are centraldifferenced at the cell interfaces using the primitive variables of the adjacent cells and transport properties based on the arithmetic mean of the static temperatures in the adjacent cells. The inviscid flux vectors are computed at the cell interfaces so as to effect a secondorder accurate upwind-difference using the MUSCL-type interpolation and difference schemes described below.

Interpolation. Spekreijse's interpolation ${ }^{16}$ of the primitive variables $(\rho, \underline{u}, p)$ with Van Albada limiting ${ }^{17}$ is used to set the "left" and "right" states of the cell interfaces. Our choice of MUSCL-type ${ }^{18}$ interpolation to effect a second-order accurate, monotonicity preserving scheme, was influenced by the work of Anderson et al. ${ }^{19}$ As implemented by Orkwis and $\mathrm{McRae}{ }^{20}$, the primitive variables are set (e.g., at a constant- $\eta$ interface) by

$$
\begin{aligned}
& u_{i+\frac{1}{2}}^{L}=u_{1}+\frac{1}{2} \frac{\left(2 d b^{2}+\delta^{2}\right) d a+\left(d a^{2}+2 \delta^{2}\right) d b}{2 d b^{2}-d a d b+2 d a^{2}+3 \delta^{2}} \\
& u_{i+\frac{1}{2}}^{R}=u_{i+1}-\frac{1}{2} \frac{\left(2 d b^{2}+\delta^{2}\right) d c+\left(d c^{2}+2 \delta^{2}\right) d b}{2 d b^{2}-d b d c+2 d c^{2}+3 \delta^{2}}
\end{aligned}
$$

where $d a=u_{1}-u_{i-1}, d b=u_{1+1}-u_{i}$, and $d c=u_{i+2}-u_{i+1}, \delta=1 \times 10^{-5}$, and where " $u$ " here represents the primitive variables. Having set the left and right states, the inviscid flux at a cell face is calculated by using the approximate Riemann solution of $\mathrm{Roe}^{3}$ or the flux-splitting scheme of Liou and Steffen ${ }^{4}$.

Roe flux difference splitting. The approximate Riemann solution of $\mathrm{Roe}^{3}$ provides an estimate of the inviscid flux at a cell face of constant- $\eta$ (for example) through

$$
\begin{aligned}
E_{i+\frac{1}{2}}^{R O \theta} & =\frac{1}{2}\left\{E\left(\underline{u}_{L}\right)+E\left(\underline{u}_{R}\right)\right\}-\frac{1}{2}|\underline{\underline{\beta}}| \delta Q \\
& =\frac{1}{2}\left(E_{L}+E_{R}\right\}-\frac{1}{2} \sum_{I=1}^{4}\left|\hat{\lambda}_{1}^{*}\right| \hat{\Sigma}_{1} \delta w_{1}
\end{aligned}
$$

where ^ denotes Roe-averaged variables", " $L$ " and "R" denote the left and right states of the Riemann problem set by the interpolation, and $E_{L}$ and $E_{R}$ are the flux vectors computed using the primitive variables $\underline{u}_{\mathrm{L}}$ and $\left.\underline{u}_{R}\right)$ of the left and right states. $\lambda_{1}^{*}$ is the $l_{\mathrm{Lh}}$ modified eigenvalue and $\underline{I}_{1}$ is the $t^{\text {h }}$ right eigenvector of the Jacobian matrix $\underline{A}=\partial E / \partial Q$ (see, for example, Hirsch et al. $\left.{ }^{21}\right)$, constructed using Roe-averaged variables, and $\delta w_{1}$ is the jump (left to right state) in the $l^{\text {th }}$ characteristic variable. The modified eigenvalues are the eigenvalues of the Jacobian $\underline{\underline{\hat{a}}-\text {-i.e., }} \boldsymbol{\lambda}_{1}=\hat{\lambda}_{2}=\hat{\tilde{U}}|\nabla \xi| / J$ and 
$\hat{\lambda}_{3,4}=\left(\hat{\tilde{U}}_{ \pm} \hat{a}\right)|\nabla \xi| / J$ (see Steger ${ }^{2}$ and his reference to Warming et al..$^{2}$ for the eigenvalues in moving generalized coordinates)-corrected using the entropy condition fix of Harten and $\mathrm{Hyman}^{24}$ to assure that expansion shocks are not admitted by the solution. The generalized coordinate formulation of Roe's flux difference splitting used in this work is that presented by Grossman and Walters ${ }^{25}$ and Walters and Thomas. ${ }^{26}$ The flux vector in the $\xi$-direction (at a cell face of constant- $\eta$ ) can be written as

$$
E_{i+\frac{1}{2}}=\frac{1}{2}\left\{E_{L}+E_{R}\right\}-\frac{1}{2} \sum_{i=1}^{4}\left|\delta E_{1}\right|
$$

where

$$
\begin{aligned}
& \left|\delta F_{2}\right|=\frac{|\nabla \xi|}{J}|\hat{\tilde{U}}|\left(\delta \rho-\frac{\delta p}{\hat{a}^{2}}\right)\left[\begin{array}{c}
\overline{1} \\
\hat{\theta} \\
\hbar-\frac{\hat{a}^{2}}{\gamma-1}
\end{array}\right] \\
& \left|\delta E_{2}\right|=\frac{|\nabla \xi|}{J}|\hat{\tilde{U}}| \hat{\rho}\left[\begin{array}{c}
\delta u-\xi_{x} \delta u^{\xi} \\
\delta v-\xi_{y} \delta u^{\xi} \\
0 \delta u+\theta \delta v-a^{\xi} \delta u^{\xi}
\end{array}\right]
\end{aligned}
$$

and

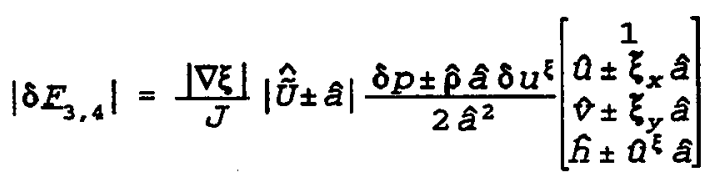

where $u^{\xi}=\tilde{U}+\nabla \xi \cdot \dot{\boldsymbol{x}}=\tilde{U}-\xi_{t}$, the jumps are from the left to right states (e.g., $\delta u=u_{R}-u_{L}$ ), and where the normalized metrics are those of the cell surface, described earlier. Note that $\delta u^{\xi}=\delta \tilde{U}$ for the case where the grid velocity $\dot{x}$ is uniform in space, as is the case in the wave rotor. The G-vector (e.g., $G_{j+\frac{1}{2}}$ ) is computed at the cell faces composed of constant- $\xi$ lines in an analogous fashion.

We note that the grid motion influences the above formulation in that the contravariant velocity components ( $\tilde{U}$ and $\tilde{V}$ ) are those relative to the moving grid and therefore the eigenvalues are affected by the grid motion. Because the Jacobian $\underline{\underline{A}}$ is given by $\underline{\underline{A}}=\xi_{t} \underline{\underline{I}}+\underline{\underline{A^{\prime}}}$ where $\underline{A}^{\prime}$ is the Jacobian $\partial E / \partial Q$ for a stationary grid system, the eigenvectors of the Jacobian $\underline{A}^{\prime}$ of the stationary system are also the eigenvectors of the Jacobian $\underline{\underline{A}}$ of the moving grid system; i.e., the Roe dissipation term $\left(\frac{1}{2}|\underline{\underline{\hat{\beta}}}| \delta \Omega\right)$ on the moving grid system can be decomposed into the standard (fixed grid system) right eigenvectors and jumps in characteristic variables, as done above, and the eigenvalues are shifted by the grid speed.
AUSM flux vector splitting. As an alternative to the computationally expensive Roe scheme, the Advection Upstream Splitting Method (AUSM) of Liou and Steffen ${ }^{4}$ (see also Liou') has also been investigated to set the inviscid flux vector. Accommodating the grid motion, and in generalized coordinates, the AUSM splitting is used in this work by calculating the inviscid flux (e.g., here at a cell face composed of a constant- $\eta$ line and using the notation of Lious) as

$$
E_{i+\frac{1}{2}}^{X N S P}=\frac{|\nabla \xi|}{J}\left\{\frac{1}{2} \tilde{U}_{\frac{1}{2}}\left(\Phi_{L}^{C}+\Phi_{R}\right)-\frac{1}{2}\left|\tilde{U}_{\frac{1}{2}}\right|\left(\Phi_{R}^{C}-\Phi_{L}^{C}\right)+E_{\frac{1}{2}}\right\}
$$

where " $L$ " and " $R$ " again denote the left and right states respectively and where

$$
\Phi^{c} \equiv\left[\begin{array}{c}
\rho \\
\rho u \\
\rho V \\
\rho h
\end{array}\right] \text { and } R_{\frac{2}{2}} \equiv\left[\begin{array}{c}
0 \\
\xi_{x} p_{\frac{1}{2}} \\
\xi_{y} p_{\frac{1}{2}} \\
-\xi_{t} p_{\frac{1}{2}}
\end{array}\right]
$$

given that $\tilde{U}_{\frac{1}{2}}=\tilde{U}_{L}^{+}+\tilde{U}_{R}^{-}$and $p_{\frac{1}{2}}=p_{L}^{+}+p_{R}^{-}$. Defining the local contravariant Mach number of a given state as $\tilde{M}=\tilde{U} / a$, where a is the local speed of sound, the $\tilde{U}_{\frac{1}{2}}$ and $p_{\frac{1}{2}}$ are given by

$$
\tilde{U}^{ \pm}= \begin{cases}\frac{a}{2}(\tilde{M} \pm|\tilde{M}|) & \text { if }|\tilde{M}| \geq 1 \\ \pm \frac{a}{4}(\tilde{M} \pm 1)^{2} & \text { if }|\tilde{M}|<1\end{cases}
$$

and

$$
p^{ \pm}=\left\{\begin{array}{cl}
\frac{p}{2}(1 \pm \tilde{M} /|\tilde{M}|) & \text { if }|\tilde{M}| \geq 1 \\
\frac{p}{4}(\tilde{M} \pm 1)^{2}(2 \mp \tilde{M}) & \text { if }|\tilde{M}|<1 .
\end{array} .\right.
$$

The AUSM scheme effectively sets the convective part of the inviscid flux at the cell surface to be $\tilde{U}_{\frac{1}{2}} \Phi_{L}^{c}$ if $\tilde{U}_{\frac{1}{2}} \geq 0$ or $\tilde{U}_{\frac{1}{2}} \Phi_{R}^{C}$ otherwise. Liou and Steffen ${ }^{4}$ note that the third-order pressure splitting used in the subsonic region can cause small oscillations immediately (one cell) downstream of a shock. A simpler first order expansion can be used in the subsonic region ${ }^{4}: p^{ \pm}=p(1 \pm \tilde{M}) / 2$. The third-order pressure splitting is used in the present work. Liou and Steffen also point out that the AUSM scheme, like van Leer's flux splitting scheme, uses a scalar coefficient $\left(\tilde{U}_{\frac{1}{2}}\right)$ and therefore requires only $O(n)$ operations for an $\mathbf{n}$-dimensional flux vector as compared to $O\left(n^{2}\right)$ operations in the Roe scheme because of the matrix coefficient. For example in a computation in this present work of Mach 2 steady inviscid flow over a 10\% bump, 1000 iterations with the AUSM scheme required 
0.70 time units as compared to the 1.0 time units required by the Roe scheme with the entropy fix. The AUSM scheme shows promise of computational efficiency while maintaining accuracy.

The overall thin-layer Navier-Stokes solver is spatially and temporally second-order accurate. It has been demonstrated for both the Roe flux difference splitting (see, for example, van Leer et al. ${ }^{27}$ and von Lavante ${ }^{28}$ ) and the AUSM splitting scheme (see Liou')that the combination of the upwind treatment of the inviscid flux terms and the central-difference of the diffusion terms accurately models steady viscous flow fields. Here we apply these methods to the unsteady wave rotor viscous flows.

\section{Boundary and Interface Conditions}

The unsteady wave rotor flow field has been modeled using both a single-passage solver and a multi-block solver.

Single-passage. In the single-passage computations, the flow field is computed in the frame of reference of the moving passage and moving boundary conditions are applied at the ends of the single computational block as shown schematically in Figure 3. In the single-passage computations reported here, the inflow boundary is set by completely specifying the primitive variables at the inflow plane (State a) and by requiring that the expansion fan emitted upstream, from the passage, be infinitely weak (cf. Eidelman ${ }^{29}$ ); i.e., the primitive variables at the phantom point $(i=0)$ are those of State a. The $v$ component of velocity is set to the rotor speed. At an outflow boundary (at the right end of the passage shown in Figure 3), the static pressure is specified at the outflow plane (state $b$ ) and the invariance of $R^{+}=2 a /(\gamma-1)+u$ and $p / \rho^{\gamma}$ across the fan is used to set the phantom point (implying that the emitted contact and shock are infinitely weak), and $v$ is linearly extrapolated from the interior. The effects of the gradual opening of the rotor passages to the inflow or outflow ports is simulated, after Eidelman ${ }^{29}$, by gradually replacing the wall boundary conditions described next by the appropriate inflow or outflow conditions, based on the rotor linear speed.

At constant $-\eta$ walls, $\partial p / \partial n=0$, where $n$ is the local normal to the wall, and $\mathrm{V}=0$ is enforced within the inviscid flux vectors. At constant $-\xi$ walls, $\partial p / \partial n=0$ is enforced by using phantom points in the wall; e.g., $\tilde{U}_{0}=-\tilde{U}_{1}$ and $p_{0}=p_{1}$ are required and additionally, $\tilde{V}_{0}=\tilde{V}_{1}$ is required for an inviscid flow. For viscous calculations, $\partial T / \partial n=0$ and $\tilde{U}=\tilde{V}=0$ are also required at all the walls and these conditions are enforced by using phantom points.

Multi-block. In the multi-block calculations, the phantom points to the left of the inlet port inflow plane are set by specifying the total.pressure and total temperature and by extrapolating the left-running Riemann invariant $R^{-}=2 a /(\gamma-1)-u_{\xi}$ (where $u_{\xi}$ is the covariant $\xi$-velocity component) from the first interior point. The downstream boundary of the inlet port and the upstream boundary of the rotor passages currently within the span of the inlet port coincide as shown in Figure 2. This surface is treated as an interface and is updated at the end of a complete time step, after incrementally advancing the grid as appropriate for the current time step and the rotor linear speed. The phantom point at the downstream boundary of the inlet port is set using linear interpolation of the conserved variables on the current rotor solution while the phantom point at the inlet of the rotor passages is set using linear interpolation of the conserved variables on the current inlet port solution. Outside of the inlet port span, the phantom points at the left end of the rotor passages are set to effect the appropriate wall boundary conditions as described above for the single-passage calculations.

At the right-hand end of a rotor passage, the phantom points are set to effect the constant- $\xi$ wall boundary conditions for those points outside of an outlet port span. For simulations in which outlet ports are included, the phantom points exposed to the outlet port flow are set as in the single passage calculations; i.e., a uniform static pressure is specified at the outlet boundary and the invariance of the $R^{+}$and $p / p^{\gamma}$ across the fan that is assumed to enter the passage is used to set the phantom point, and the $v$ velocity component is linearly extrapolated from the interior; however, if the resulting $u$ velocity component at the phantom point is negative, indicating local inflow rather than outflow, the phantom point stagnation temperature is then specified, the $u$ velocity component is set to zero, and the mass density is calculated based on the local temperature and the same specified back pressure.

\section{Model Validation}

\section{Steady Flow}

After MacCormack and Lomax ${ }^{30}$, the ability of the solver to model accurately a stationary oblique shock/laminar boundary layer interaction is evaluated by comparing computed results of the present work with the experimental data of Hakkinen et al. ${ }^{31}$ and calculated results of Liou and Steffen ${ }^{4}$ using their AUSM scheme. Figures $4 \mathrm{a}$ and $4 \mathrm{~b}$ show comparison of the normalized static pressure $\left(p_{\text {wall }} / p_{\infty, \text { in }}\right)$ and the skin friction coefficient $\left(C_{f} \equiv 2 \tau_{w} /\left(\rho u^{2}\right)_{\omega, \text { in }}\right)$ of a Mach 2 flow along a flat plate (with a leading edge at $x=1$ ). Both the AUSM flux-splitting and Roe flux-differencing schemes as implemented in the present work are compared. The present computations were performed on 
a $121 \times 101$ stretched grid. Liou and Steffen resolved the flow field with a $75 \times 65$ stretched grid. A 32-degree oblique shock interacts with the laminar boundary layer at $\mathrm{x}=2$ where the Reynolds number is $2.96 \times 10^{5}$, and causes separation. The computed results using the Roe and AUSM schemes as implemented in the present study are virtually identical. The static pressure distributions predicted by both the Roe and AUSM schemes in this present study agree very well with the experimental data of Hakkinen et al. ${ }^{31}$ The static pressure predicted by Liou and Steffen using their AUSM scheme is low in the recirculation region (as discussed by Liou and Steffen ${ }^{4}$ ). The skin friction coefficient predicted by the present method is lower than the experimental data over much of the plate. The results of Liou and Steffen's calculation better predict the extent of the recirculation zone indicated by the skin friction coefficient. Qualitatively, the CFD predictions of the present study and experimental data of Hakkinen et al. match well.

\section{Unsteady Flow}

The wave rotor passage gradual opening problems considered by Eidelman ${ }^{29}$ have been repeated as a form of CFD validation. Eidelman used a second-order extension of the Godunov method (see Eidelman et al..$^{32}$ for details of their model). Figure 5 provides a comparison of snapshots in time of the static pressure contours reported by Eidelman ${ }^{29}$ and computed here as a single passage calculation. The problem models a single wave rotor passage moving downward in Figure 5 at 200 $\mathrm{m} / \mathrm{s}$. The present calculations use the AUSM fluxsplitting scheme and are performed on a uniform grid of $121 \times 25$ points which provided the same resolution as that used by Eidelman. The conditions in the inlet port/passage interface are $p_{\text {in }}=0.182 \mathrm{MPa}(1.80 \mathrm{~atm})$, $u_{\text {in }}=150 \mathrm{~m} / \mathrm{s}, v_{\text {in }}=0$, and $\rho_{\text {in }}=1.81 \mathrm{~kg} / \mathrm{m}^{3}$, and the conditions on board the passage prior to opening are $p_{0}$ $=0.103 \mathrm{MPa}(1.0 \mathrm{~atm}), \rho_{0}=1.2 \mathrm{~kg} / \mathrm{m}^{3}$, and $u_{0}=0$. These conditions assure that there is an insignificant density change across the driver gas/driven gas contact. The passage itself does not move in Eidelman's problems but rather the gradual opening of the passage to the inlet port flow is simulated by gradually replacing the left wall boundary condition with the inlet port flow conditions in accordance with the rotor speed of $200 \mathrm{~m} / \mathrm{s}$ such that the passage is fully open at $0.1 \mathrm{~ms}$. The time sequence compared in Figure 5 indicates that the two models agree well. The flow field appears to better resolved in the current study. Both models clearly show the development of the compression wave from an initial "spherical" wave, through reflections of the pressure wave first off the trailing (upper) passage wall (evident at $t=0.086 \mathrm{~ms}$ ), then the off the leading wall (evident at $t=0.127 \mathrm{~ms}$ and $t=0.168 \mathrm{~ms}$ ), and finally off the trailing wall again (evident at $t=0.252 \mathrm{~ms}$ ). A discussion of the reflection of the compression wave and the highly rotational flow that results from the gradual, rather than instantaneous passage opening can be found in Eidelman's paper. ${ }^{29}$

The single-passage problem was extended to model the shock/contact interaction and shock/boundary layer interactions by including viscous effects and by allowing the initially rightward running shock wave to reflect off the right-hand wall. The inlet port/passage interface conditions in this computation are $\mathrm{p}_{\text {in }}=0.289 \mathrm{MPa}(2.85$ atm), $u_{\text {in }}=283 \mathrm{~m} / \mathrm{s}$, and $\rho_{\text {in }}=4 \mathrm{~kg} / \mathrm{m}^{3}$, and the rotor speed in this calculation is $250 \mathrm{~m} / \mathrm{s}$. The on-board passage conditions are the same as in the inviscid case. These conditions are those of a second case considered by Eidelman ${ }^{29}$ and assure that the driver gas/driven gas contact discontinuity is significant so that the shock/contact interaction can be studied. The calculations were performed on a 121 × 61 stretched grid using the AUSM flux-splitting. Figure 6 shows velocity vectors and density contours at a time when the reflected shock, apparent at the left side of the picture, has moved through the contact discontinuity. (Note that in this picture the left wall was gradually withdrawn in the direction opposite of that in the inviscid problem above.) The strong vortical structure following the shock/contact interaction is clearly visible. Note that the highly rotational flow leads to mixing between the driver and driven gases and is a loss mechanism in the wave rotor. At the position of the reflected shock, a strong unsteady shock/boundary layer interaction is evident. The boundary layer fluid into which the shock moves lacks the momentum to negotiate the shock pressure rise so that separation occurs, a recirculation region is formed, and the upstream boundary layer is lifted, resulting in turning of the (relative) supersonic free stream through an oblique shock stem. The flow is then turned again to the axial direction via another oblique shock stem. The reflected shock is therefore a running lambda-shock. From Figure 6 it is apparent that the effective distance in which the upstream fluid is brought to rest is the length of the recirculation region.

\section{Model Inlet Port Simulation}

Multi-block calculations have been used to study the influence of inlet port corner rounding and of profiling the leading passage wall on the shape and behavior of the contact discontinuity established by the charging process at an inlet port. More detailed results of the study have been reported in Ref. 9. A brief description of the model inlet port simulation is presented below along with example results showing the evolution of the contact discontinuity during the rotor charging process.

The inlet port opening of this simulation spans five rotor passages, each of which has a blade-to-blade width of 0.1 units and a length of 1.0 units. The inlet port walls are each slanted at 30-degrees (off the horizontal) so that the mean $y$-component of velocity nearly matches 
the rotor linear speed. Seven rotor passages are included in a calculation at any given time. As a virgin passage enters the computational domain, the most advanced passage is discarded. At the inlet port inflow plane, the stagnation pressure and temperature are specified as $\mathbf{3 . 0}$ and 1.0, respectively, in nondimensional units. These conditions are kept constant in space and time. The onboard virgin passage stagnation pressure is 1.0 , the onboard stagnation temperature is $\mathbf{1 . 0}$, and the relative velocity is zero. The wheel speed is selected so that the initially rightward running shock wave in a given passage reflects off the right-hand wall and arrives back at the inlet port/passage interface plane as that passage is closing. The right-hand side of the rotor passages move past a fixed wall everywhere in the example simulation. The inlet port grid is $56 \times 101$ points and each of the seven passage grids has $106 \times 31$ points, for the inviscid flow shown here. The AUSM flux-splitting scheme was used in these computations.

The initial conditions were uniform Mach number (0.5) in the inlet port and virgin passage conditions in each passage. The inlet stagnation conditions and virgin passage conditions are constant and therefore the unsteady flow field reaches temporal periodicity after a large number of rotor passages have traversed the inlet port. The unsteady flow field typically begins to assume periodic behavior in about three times the time it takes a given passage to traverse the inlet port span (e.g., three thousand iterations). True periodicity however requires longer running time and is dependent on the grid resolution. Ten to fifteen thousand iterations were required to reach periodicity in these inviscid flow simulations.

\section{Sharp Inlet Port Lip}

Figure 7 shows density contours for an inlet port with a sharp cornered lip (no rounding). The passage is 66 percent open at the time shown. A rightward running compression wave in the first (bottom) passage is seen to steepen quickly into a rightward running shock wave (as seen in the second passage). Simultaneously an expansion wave enters the inlet port region. The rightward running shock wave compresses the on-board driven gas, setting it into motion and allowing the driver gas to enter the passage. The contact discontinuity moves with the local fluid velocity. Due to the sharp corner inlet lip and the asymmetry of the gradual opening process, the driver fluid is initially (see the first passage at the bottom of Figure 7) concentrated along the leading wall of the passage. Mach contours (not shown here) show that the driver fluid behind the most forward part of the interface is supersonic and is running faster than the subsonic driven gas by which it travels. There is large vorticity at the contact, apparent in the subsequent tumbling of the interface as seen in the first, second, and third passages. Note that a part of the driven gas is "captured" by the tumbling interface.
The initially rightward running shock reflects off the right wall and recompresses the driven gas, bringing the driven gas to rest (see the third passage). The leftward running reflected shock then interacts with the contact discontinuity as seen in the fourth passage. Note that any protuberance in the contact is exaggerated by the shock/contact interaction. The reflected shock further compresses the driver fluid (see the fifth passage). The reflected shock returns to the inlet plane and is emitted from the passage into the inlet port as the passage closes. The emitted shock wave is visible in the inlet port region shown in Figure 7. This fluid leaving the sixth passage is accelerated around the passage wall and enters the fifth passage. The flow in the inlet port is unsteady due to the emitted shock wave and the leftward moving expansion wave created as new passages open to the inlet port.

\section{Inlet Lip Rounding}

Figure 8 shows the density contour plots for a wave rotor inlet port with lip rounding at the same time as the previous example. Comparing Figures 7 and 8 shows that the corner rounding has a large effect on the behavior of the contact discontinuity. In this case the leading lip of the contact discontinuity is located at the trailing wall of the passage (see the first, second, and third passages). The contact discontinuity curls in the direction opposite that of the sharp lip case after the reflected shock/contact interaction. Due to the asymmetry of the gradual passage opening process, a portion of the driven fluid is again captured by the driver fluid; however, the corner rounding appears to have significantly reduced the amount of driven fluid captured, suggesting that comer rounding can help control driver/driven gas mixing.

\section{NASA LeRC Divider Cycle Simulation}

The medium pressure inlet port and the high pressure outlet port region of the NASA LeRC three-port divider cycle experiment (see Wilson and Fronek ${ }^{6}$ ) has been modeled. In a three-port divider cycle, typically a small fraction (e.g., 0.3) of the medium pressure stream that enters through the inlet port is compressed to a higher stagnation pressure $\left(\mathrm{e} . \mathrm{g}, \tilde{p}_{\text {high }} / \tilde{p}_{\text {medium }} \simeq 1.3\right)$ and is then exhausted into the high pressure outlet port. The remainder of the stream is expanded later in the cycle to a lower stagnation pressure (e.g., $\tilde{p}_{\text {low }} / \tilde{p}_{\text {modium }}=0.78$ ) and is exhausted to the low pressure outlet port (see, for example, Kentfield ${ }^{\prime}$ ). In this simulation, the medium pressure port and 39 rotor passages that are near the medium pressure inlet port or the high pressure outlet port are computed with individual blocks. This portion of the NASA LeRC divider cycle is similar to the model inlet port problem described earlier (and shown schematically in Figure 2); however, the high pressure outlet port on the right end of the rotor allows the twicecompressed driven flow to leave the rotor passage. The outlet port is represented here by a uniform static 
pressure surface. A more detailed discussion of this simulation has been reported in Ref. 9.

Two viscous calculations for the divider cycle were performed using Roe's scheme. In the first, the virgin passage flow is set to zero relative velocity and the onboard pressure and temperature are set to best estimate conditions based on experimental data. In the second calculation, the virgin passage conditions are a 2-D construction of the passage conditions just prior to opening to the inlet port based on 1-D computations of the full divider cycle. ${ }^{8}$

Experimentally measured quantities (private communication with J. Wilson at NASA LeRC, 1993) are specified at the inlet and exit ports. The inlet port lip of the NASA experiment is rounded in order to prevent flow separation as the fluid enters the moving virgin passage. The inlet port spans 27 passages at a given time. The walls of the inlet port are parallel and are oriented at 22-degrees off the horizontal. A $20.3 \mathrm{~cm}$ ( $8.0 \mathrm{in}$.) long by $17.1 \mathrm{~cm}(6.75 \mathrm{in}$.) wide (span) portion of the inlet port is resolved by a $81 \times 101$ stretched grid. The rotor passages are $0.64 \mathrm{~cm}(0.25 \mathrm{in}$.) wide by 45.7 $\mathrm{cm}$ (18.0 in.) long and each of the 39 rotor passages is resolved by a $151 \times 15$ stretched grid. The average passage radius is $15.2 \mathrm{~cm}(6.0 \mathrm{in}$.) and the rotor spins at 3924 rpm.

Figure 9 shows static density contours at a point when the first passage is 66 percent open to the inlet port. The calculation is performed using the 1-D CFD-based virgin passage initial conditions mentioned above. The initial rightward running compression shock is mistimed and arrives late at the right-hand end of the passage; i.e., the passage has already opened to the outlet port flow, flow reversal occurs, and fluid enters the passage from the outlet port until the shock arrives. The shock then reflects not off the wall but off of the fluid entering from the outlet port. The late arrival of the shock is experimentally verified by a pressure transducer trace. Behind the rightward running shock is a rightward moving region of low density fluid that entered the passage earlier by leakage from the rotor casing. This low density fluid is evident in this 2-D calculation only because it is included in the virgin passage density distribution. Following this low density fluid is the contact discontinuity. The reflected shock passes through the contact, resulting in stretching of the interface, consistent with the inviscid and viscous results of the previous section. The reflected shock is mistimed and arrives at the inlet port/passage interface well before the port closes, consistent with experimental measurements.

A rightward running expansion wave is generated as the passages exposed to the inlet port close. Likewise, a weak hammer shock is created at the right-hand end as passages emptying into the outlet port close. The effect of the corner rounding is evident in the low density region just above the rounding.

Figure 10 compares the effectively time-averaged experimental static pressure distribution (private communication with J. Wilson at NASA LeRC, 1993) along the inlet port circumference (here normalized to one) with results from the two computations mentioned above. Qualitatively the CFD and experimental measurements agree fairly well; however, in both CFD calculations the static pressure predictions are low, indicating that the flow is moving relatively faster in the CFD solution than in the experiment. Indeed Figure 11 shows that the CFD predictions of the total velocity are significantly higher than the experimentally measured values. It is noted that the effect of blockage due to the thickness of the passage walls, comprising nearly $16 \%$ of the total port flow area, is not accounted for in the calculations for which infinitely thin walls have been assumed. Accounting for this blockage should improve agreement. Figure 12 compares the CFD predictions and the experimental measurements of the flow angle ( $\tan ^{-}$ $\left.{ }^{1}(v / u)\right)$ circumferentially along the inlet port. The predicted flow angles are consistently higher than the measured flow angles; however, the qualitative behavior is effectively modeled. Finally, note that the results of the two CFD predictions qualitatively agree, suggesting that the discrepancy between the flow fields is due largely to differences in the level of the on-board pressure in the virgin passage rather than due to the band of leakage fluid. This observation gives confidence in the ability to accurately simulate the qualitative behavior of the medium and high pressure port flow dynamics using simple, best estimate, quiescent flow conditions in the virgin passage. The agreement between the experimental data and the CFD prediction of the nearly steady port flow and between the CFD-predicted wave timing and that of the experiment, give confidence that the solver is doing a good job predicting the wave rotor flow dynamics in the vicinity of the medium and high pressure ports.

\section{Summary}

A 2-D, multi-block, thin-layer Navier-Stokes solver, developed to simulate wave rotor flow dynamics, has been described. The governing equations in finitevolume form are integrated in time using a four-stage Runge-Kutta scheme. MUSCL-type interpolation sets the "left" and "right" states used by either the Roe's flux difference splitting scheme or the AUSM flux-splitting scheme to compute second-order accurate, monotonicitypreserving, upwind-differences of the inviscid flux vectors at the finite-volume cell interfaces. The diffusion terms are central-differenced. The 2-D solver, WROTOR2D, has been validated using steady and unsteady flow problems. The AUSM scheme is found to require 0.7 times the cpu-time of the Roe scheme with entropy fix, as implemented in this work. The AUSM 
scheme promises both computational efficiency and accuracy.

A comparison of computed results, using both the Roe and AUSM schemes, with experimental data indicate that the solver accurately models stationary shock/laminar boundary layer interaction. A wave rotor passage gradual opening time problem was simulated using the AUSM scheme and computed results compared well with those of a separate study ${ }^{29}$. It is reasonable to expect that the success in predicting unsteady inviscid flow and the steady shock/boundary layer interaction problem carries over to the moving lambda-shock/boundary layer interaction problem. The unsteady shock/boundary layer interaction and the shock/contact interaction are common features of the wave rotor passage flow field, and were well resolved by the single-passage computation.

A model inlet port/rotor passage charging problem was simulated using the AUSM scheme. Key features of the unsteady flow field, including the development of the main shock wave and the contact discontinuity, the reflected shock/contact interaction, and the emission of the reflected shock from the rotor passage into the inlet port region, were well resolved. An example of the influence of inlet port lip rounding on the shape and evolution of the contact discontinuity was provided. The sources of flow unsteadiness in the inlet port region were identified from the inlet port/passage charging problem results.

Lastly, the medium pressure inlet port and high pressure outlet port portion of the NASA LeRC divider cycle experiment was simulated using the Roe scheme. The viscous flow field was computed on forty computational blocks, representing the stationary inlet port and thirty-nine moving rotor passages. Comparisons between computed results and experimental data were made. The computed wave timing is qualitatively verified by experimental pressure traces. The computed and measured static pressure, total velocity, and flow angle distributions along the inlet port qualitatively agree well.

\section{Acknowledgment}

This work was done while the first author held a National Research Council-NASA LeRC Research Associateship.

\section{References}

'Kentfield, J. A. C., "The Performance of PressureExchanger Dividers and Equalizers," Journal of Basic Engineering, Sept. 1969, pp. 361-370.
${ }^{2}$ Taussig, R. T., "Wave Rotor Turbofan Engines for Aircraft," Machinery for Direct Fluid-Fluid Energy Exchange, ed. Sladky, J. F., Jr., AD-07, presented at Winter Annual Meeting of The ASME, New Orleans, LA, Dec. 9-14, 1984, pp. 9-45.

${ }^{3}$ Roe, P. L., "Approximate Riemann Solvers, Parameter Vectors, and Difference Schemes, "J. Comp. Phys., 43, 1981, p. 357-372.

${ }^{4}$ Liou, M.-S. and Steffen, Jr., C. J., "A New Flux Splitting Scheme," NASA-TM-104404, May, 1991.

"Liou, M.-S., "On a New Class of Flux Splittings," $13^{\text {th }}$ International Conference on Numerical Methods in Fluid Dynamics," Rome, Italy, July 6-10, 1992.

"Wilson, J. and Fronek, D., "Initial Results from the NASA Lewis Wave Rotor Experiment, " AIAA-93-2521, 1993.

'Paxson, D. E. and Wilson, J., "An Improved Numerical Modelling Program for Wave Rotor Analysis," AIAA-93-0482, 1993. Also NASA-TM105915.

${ }^{8}$ Paxson, D. E., "A Comparison Between Numerically Modelled and Experimentally Measured Loss

Mechanisms in Wave Rotors," AIAA-93-2522, 1993.

'Welch, G. E., "Two-Dimensional Numerical Study of Wave Rotor Flow Dynamics," AIAA-93-2525, 1993.

${ }^{10}$ Chima, R. V., "Explicit-Multigrid Algorithm for Quasi-Three-Dimensional Viscous Flows in Turbomachinery," Journal of Propulsion and Power, 3, No. 5, Sept.-Oct., 1987, pp. 397-405.

${ }^{11}$ Keller, J. J., "Some Fundamentals of the Supercharger Comprex," Machinery for Direct FluidFluid Energy Exchange, ed. Sladky, J. F., Jr., AD-07, presented at Winter Annual Meeting of The ASME, New Orleans, LA, Dec. 9-14, 1984, pp. 47-54.

${ }^{12 B a l d w i n, ~ B . ~ S . ~ a n d ~ L o m a x, ~ H ., ~ " T h i n-L a y e r ~}$ Approximation and Algebraic Model for Separated Turbulent Flows," AIAA Paper 78-257, 1978.

${ }^{13}$ Jameson, A.; Schmidt, W., and Turkel, E., "Numerical Solutions of the Euler Equations by Finite Volume Methods using Runge-Kutta Time-Stepping," AIAA-81-1259, 1981.

${ }^{14}$ Jorgenson, P. C. E., and Chima, R. V., "An Unconditionally Stable Runge-Kutta Method for Unsteady Flows," AIAA-89-0205, 1989. Also NASA-TM-101347.

${ }^{15}$ Turkel, E. and van Leer, B., "Flux Vector Splitting and Runge-Kutta Methods for the Euler Equations," Lecture Notes in Physics, 218, Ninth International Conference on Numerical Methods in Fluid Dynamics, Springer-Verlag, New York, U.S.A., 1985, pp. 566-570.

${ }^{16}$ Spekreijse, S. P., "Multigrid Solution of the Steady Euler Equations," Ph.D. Dissertation, Centurm voor Wiskunde en Informatica, Amsterdam, 1987.

${ }^{17}$ Van Albada, G. D., van Leer, B., And Robertrs, W. W., Jr., "A Comparative Study of Computational Methods in Cosmic Gas Dynamics," Astronomy and Astrophysics, 115, No.2, 1982, pp. 263-269. 
${ }^{18}$ van Leer, B., "Towards the Ultimate Conservative

Difference Scheme. V. A Second-Order Sequel to

Godunov's Method," J. Comp. Phys., 32, 1979, pp. 101136.

${ }^{19}$ Anderson, W. K., Thomas, J. L., and van Leer, B., "Comparison of Finite Volume Flux Vector Splittings for the Euler Equations," ALAA J., 24, No. 9, September, 1986, pp. 1453-1460.

${ }^{20}$ Orkwis, P. D. and McRae, D. S., "Newton's Method Solver for the Axisymmetric Navier-Stokes Equations," ALAA J., 30, No. 6, June 1992, pp. 15071514.

${ }^{21}$ Hirsch, C., Lacor, C., and Deconinck, H., "Convection Algorithms Based on a Diagonalization Procedure for the Multidimensional Euler Equations," AIAA $8^{\text {th }}$ Computational Fluid Dynamics Conference, Honolulu, Hawaii, June 9-11, 1987, pp. 667-676.

${ }^{22}$ Steger, J. L., "Implicit Finite-Difference Simulation of Flow about Arbitrary Two-Dimensional Geometries," ALAA J., 16, No. 7, July, 1978, pp. 679-686.

${ }^{23}$ Warming, R. F., Beam, R., and Hyett, B. J., "Diagonalization and Simulations Symmetrization of the Gas-Dynamic Matrices, "Mathematics of Computations, 29, Oct. 1975, pp. 1037-1045.

${ }^{24} \mathrm{Harten}$, A. and Hyman, J. M., "Self Adjusting Grid Methods for One-Dimensional Hyperbolic Conservation Laws," J. of Comp. Phys., 50, pp. 235-269.

${ }^{25}$ Grossman, B. and Walters, R. W., "Flux-Split Algorithms for the Multi-Dimensional Euler Equations with Real Gases," Computers and Fluids. 17, No. 1, 1989, pp. 99-112

${ }^{26}$ Walters, R. W. and Thomas, J. L., "Advances in Upwind Relaxation Methods," Chapter 4 of State-of-theArt Surveys on Computational Mechanics, eds., Noor, A. K. and Oden, J. T., The American Society of Mechanical Engineers, New York, U.S.A., 1989, pp. 145-183.

${ }^{27}$ van Leer, B., Thomas, J. L., Roe, P. L., and Newsome, R. W., "A Comparison of Numerical Flux Formulas for the Euler and Navier-Stokes Equations," AIAA-87-1104, 1987.

${ }^{28}$ von Lavante, E. "Accuracy of Upwind Schemes Applied to the Navier-Stokes Equations," ALAA J., Technical Note, 28, No. 7, July, 1990, pp. 1312-1314.

${ }^{29}$ Eidelman, S., "The Problem of Gradual Opening in Wave Rotor Passages," J. Propulsion, 1, No. 1, Jan.Feb., 1985, pp. 22-28.

${ }^{30}$ MacCormack, R. W. and Lomax, H., "Numerical Solution of Compressible Viscous Flows," Ann. Rev. Fluid Mech., 11, 1979, pp. 289-316.

${ }^{31}$ Hakkinen, R. J., Greber, I., Trilling, L., and Abarbanel, S. S., "The Interaction of an Oblique Shock Wave with a Laminar Boundary Layer," NASA Memo-218-59W, 1959.

${ }^{32}$ Eidelman, S., Colella, P., and Shreeve, R. P., "Application of the Godunov Method and Its SecondOrder Extension to Cascade Flow Modeling, " ALAA J., 22, No. 11, Nov., 1984, pp. 1609-1615.

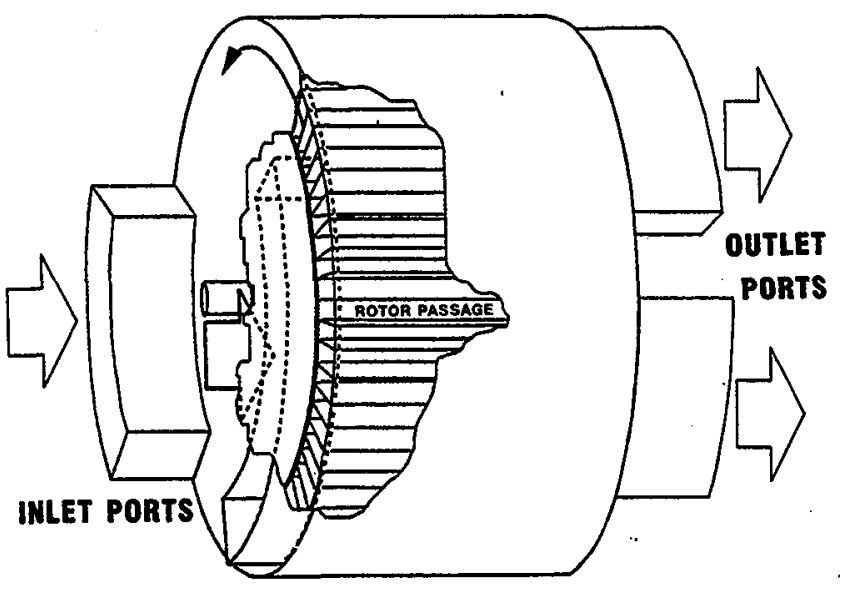

Figure 1. Schematic diagram of a four-port wave rotor (courtesy of D. E. Paxson').

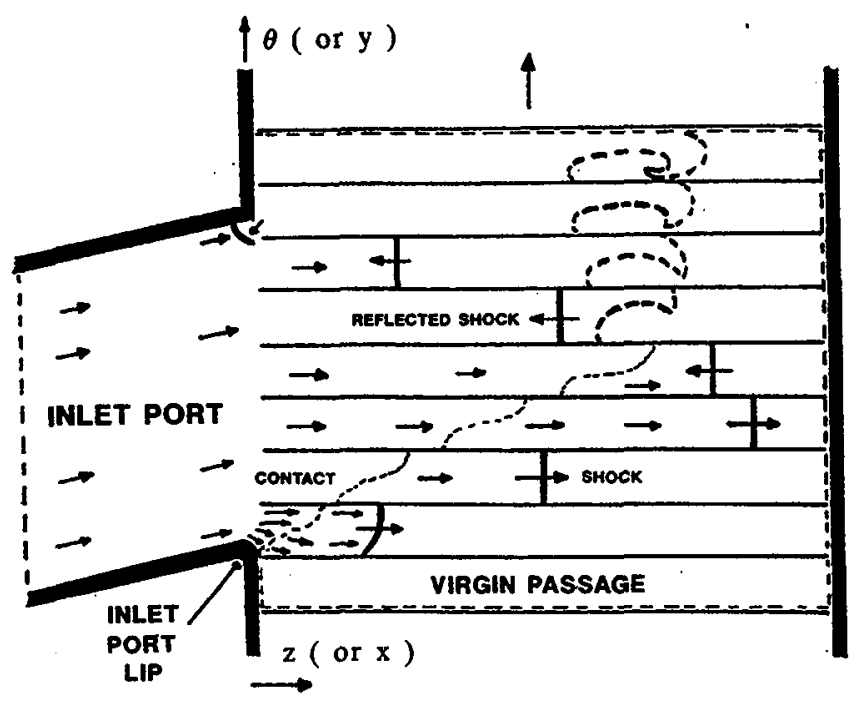

Figure 2. Model problem schematic diagram showing multiple wave rotor passages moving past inlet port. Computational domain denoted by dashed lines.

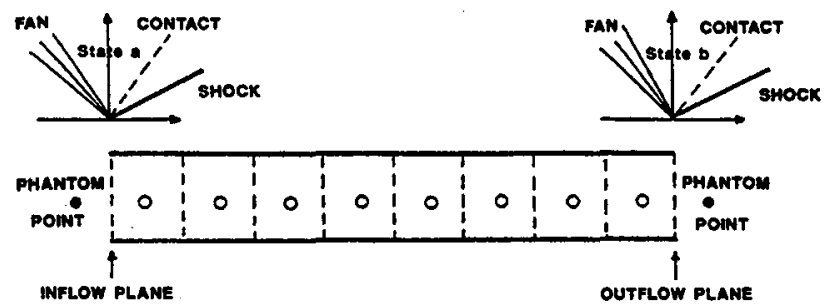

Figure 3. Single-passage inflow and outflow boundary condition diagram. 


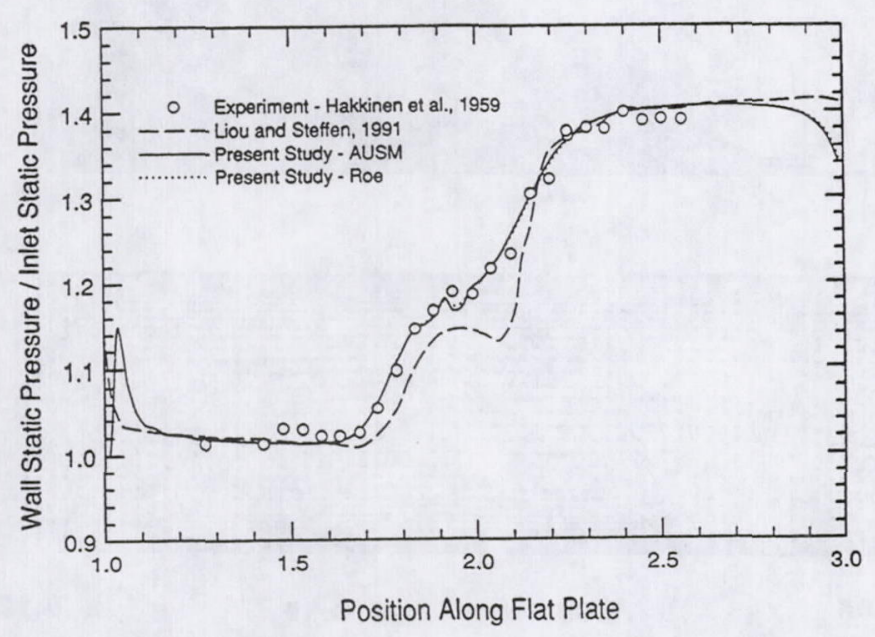

a. Normalized static pressure

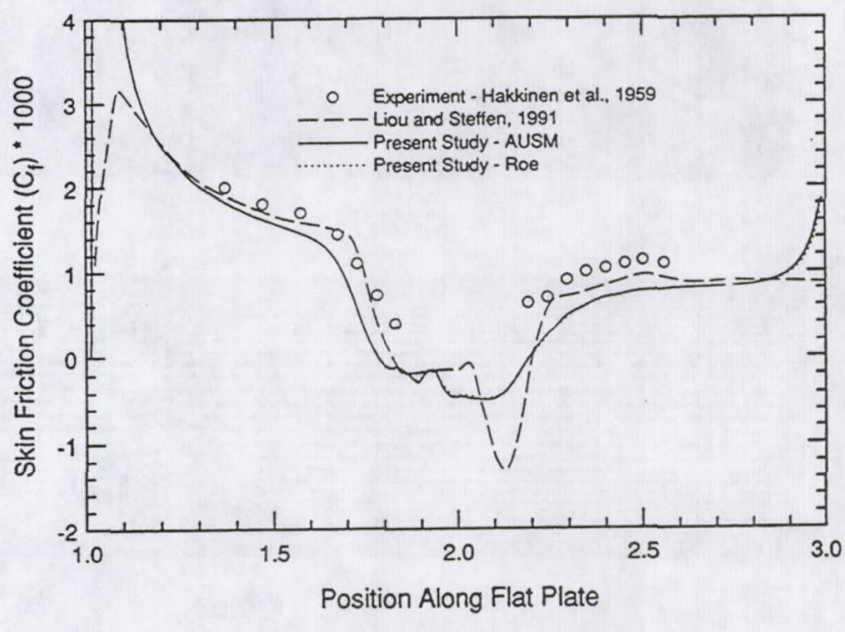

b. Skin friction coefficient

Figure 4. Comparison of oblique shock/laminar boundary layer interaction measured and computed distributions of a.) normalized static pressure, and b.) skin friction coefficient along flat plate.

-0.044 (MSEC)

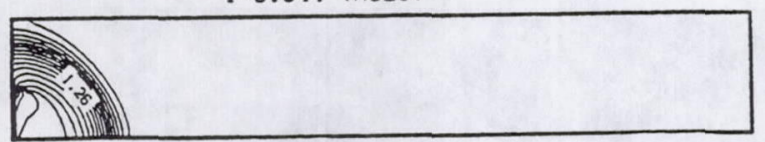

T-0.086 (MStC)

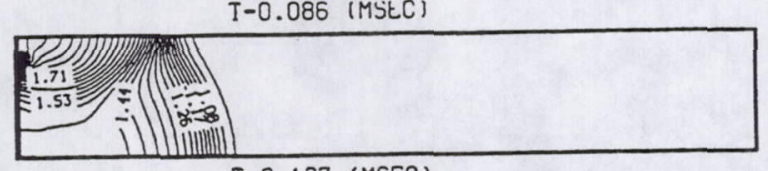

I-0.127 (MSEC)

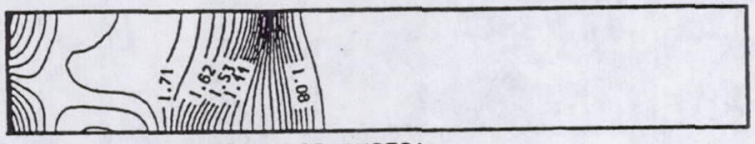

T=0.168 (MSEC)
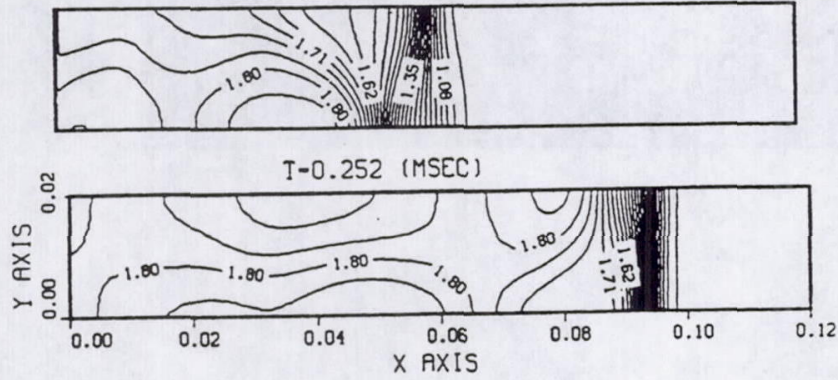

a. Eidelman's results. ${ }^{20}$
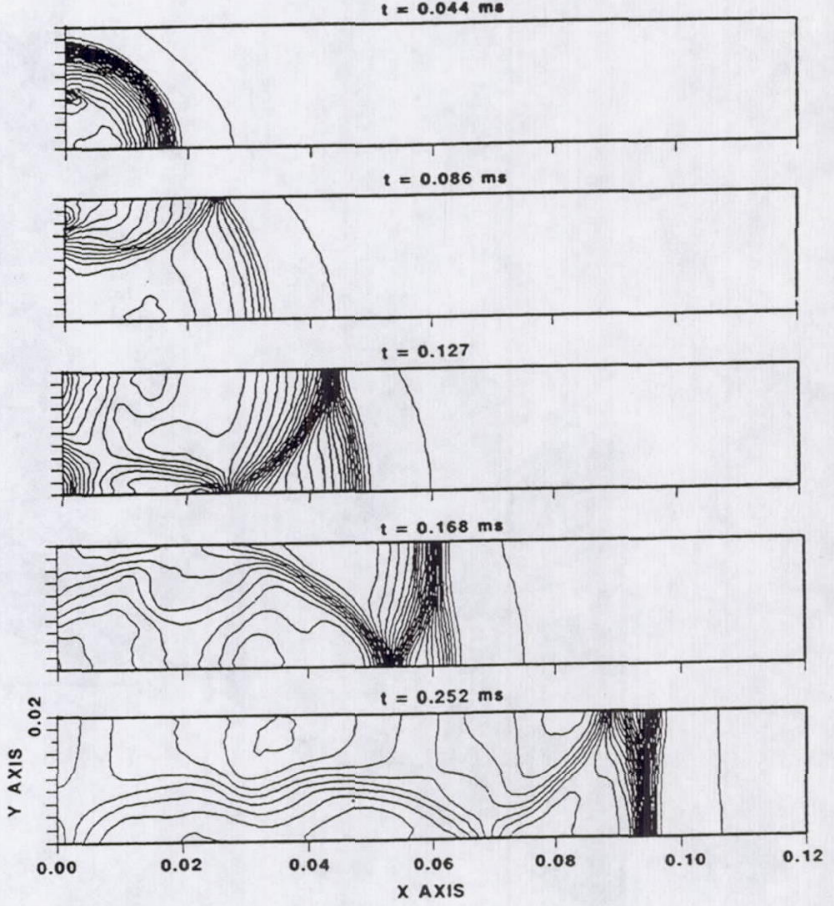

b. Present study results.

Figure 5. Comparison of computed static pressure contours by a.) Eidelman ${ }^{29}$, and b.) the present study for wave rotor gradual opening problem. 

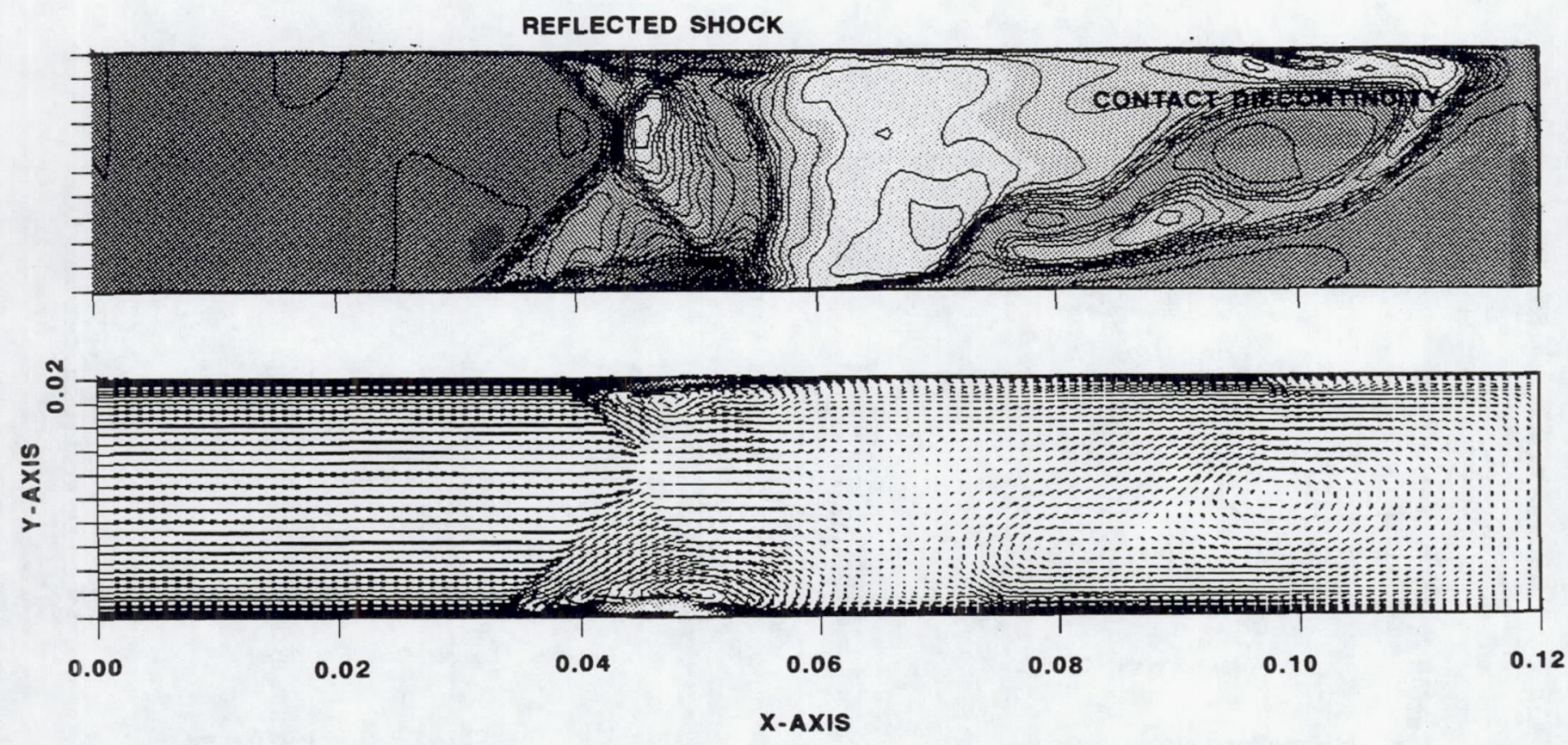

Figure 6. Density contours and velocity vectors of single passage viscous flow calculation showing the interaction of reflected shock and boundary layer and vortices generated by reflected shock and contact discontinuity interaction.

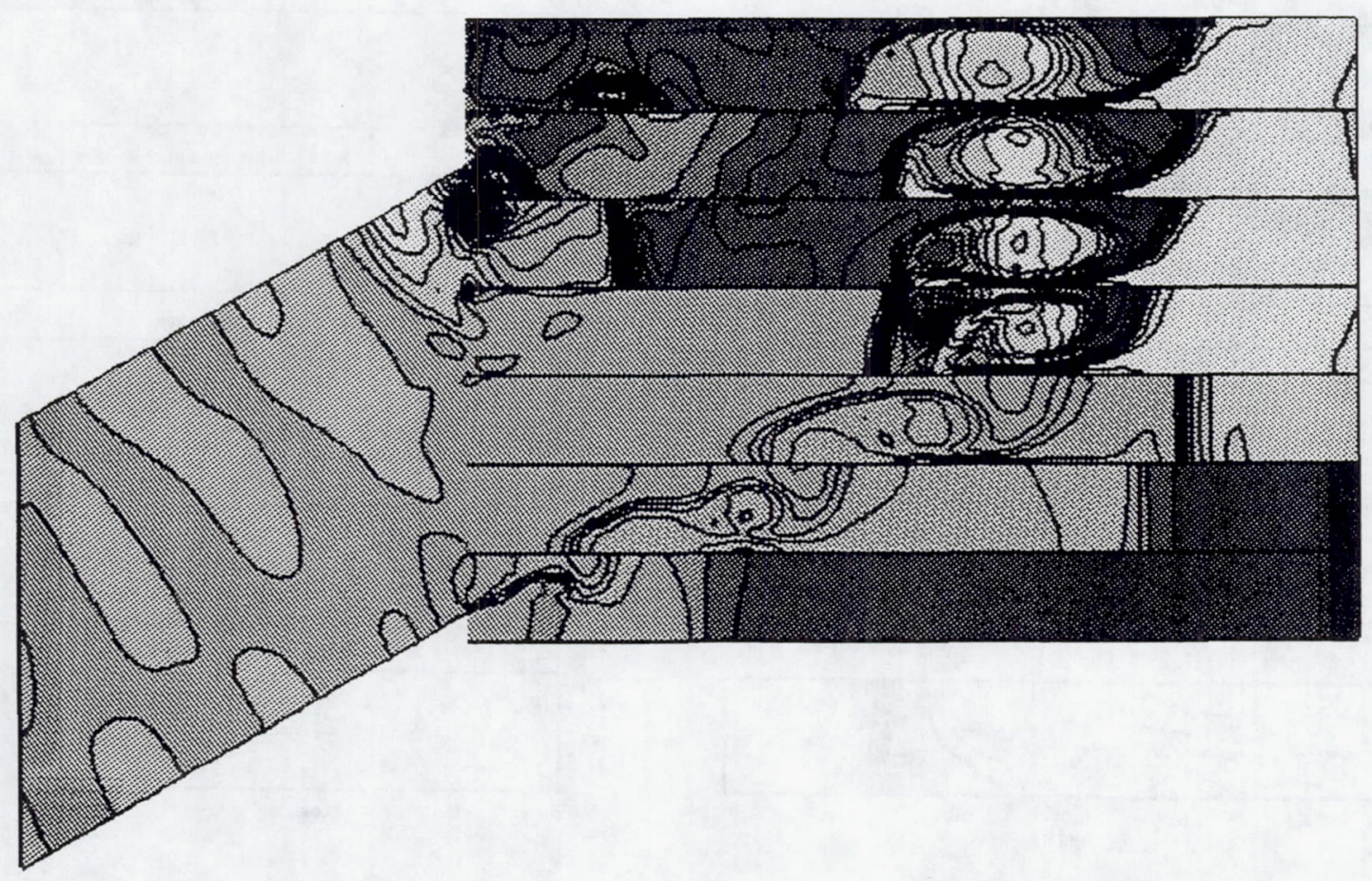

Figure 7. Computed density contours of the model inlet port/passage charging problem with sharp corner inlet port lip. 


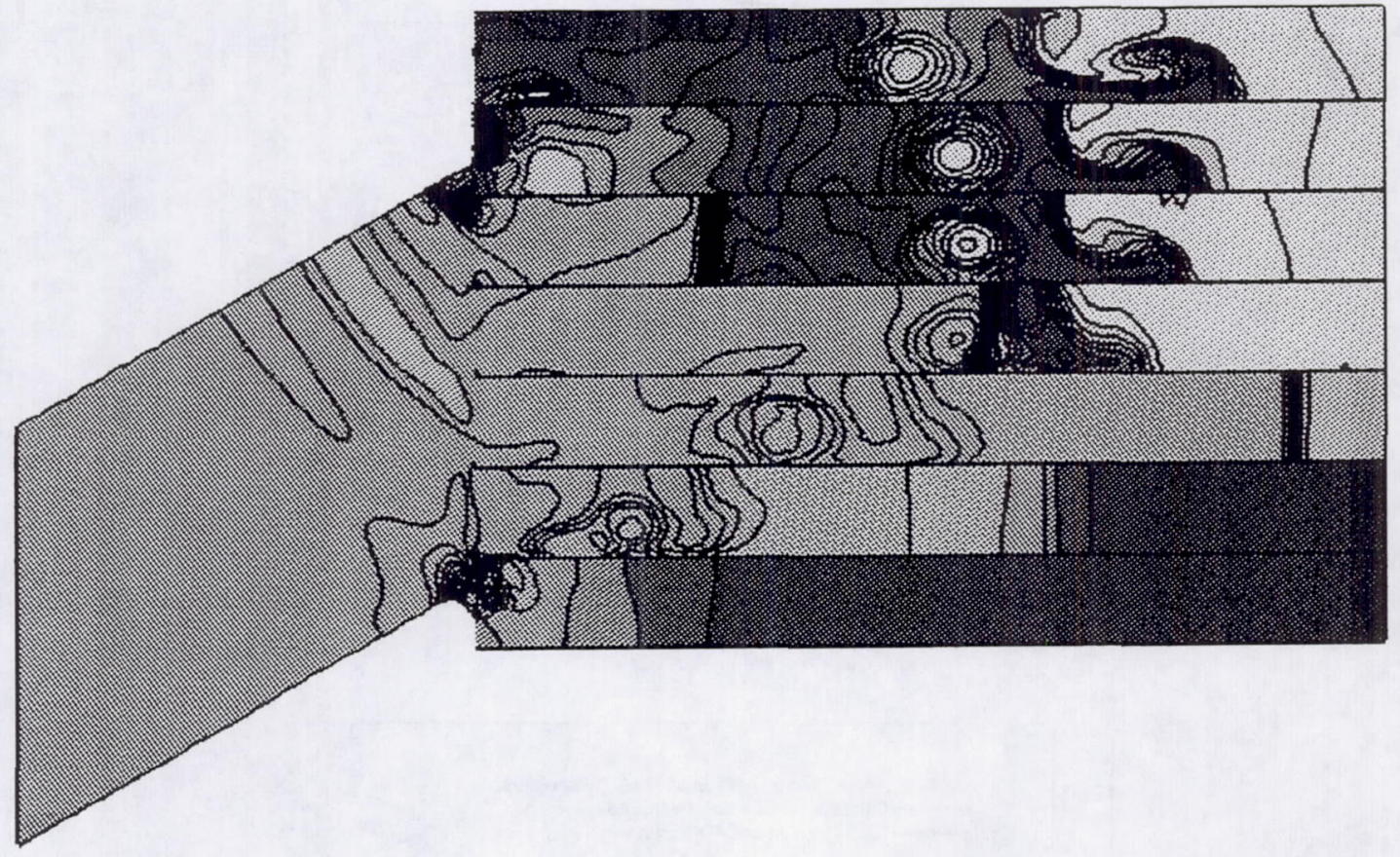

Figure 8. Computed density contours of the model inlet port/passage charging problem with rounded inlet port lip.

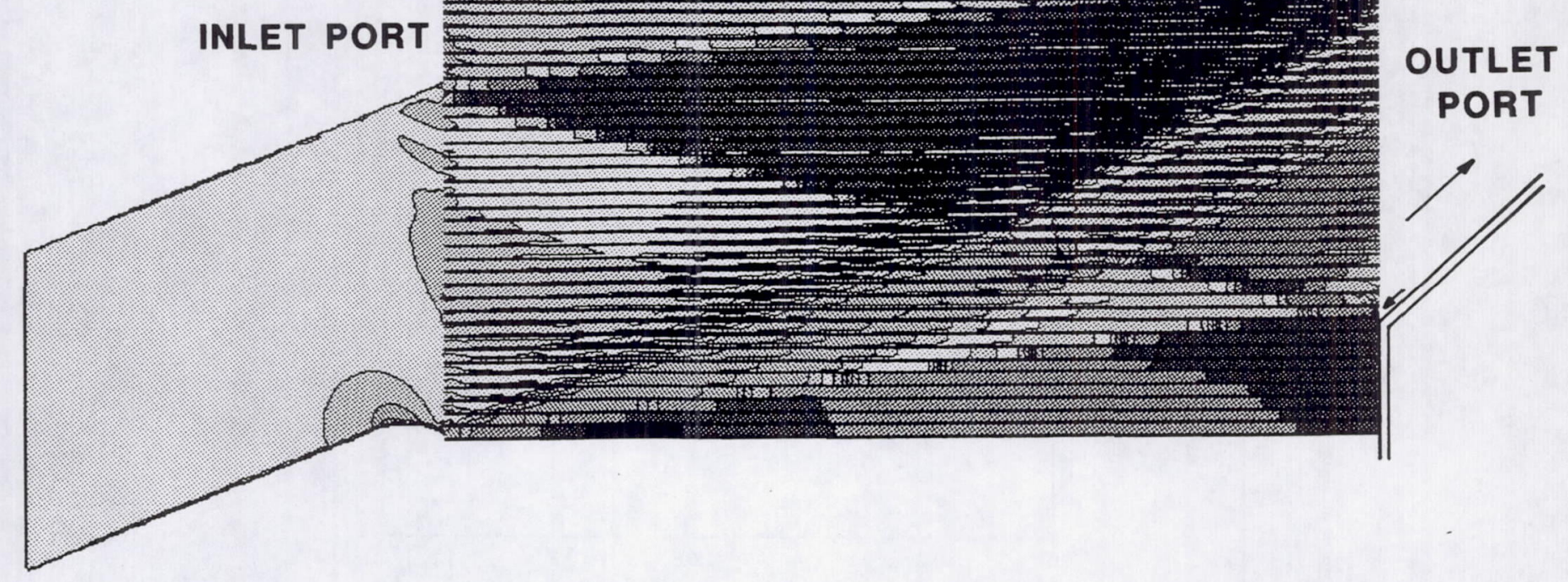

Figure 9. Computed density contours of the medium pressure inlet port and high pressure outlet port portion of the NASA LeRC divider cycle experiment. 


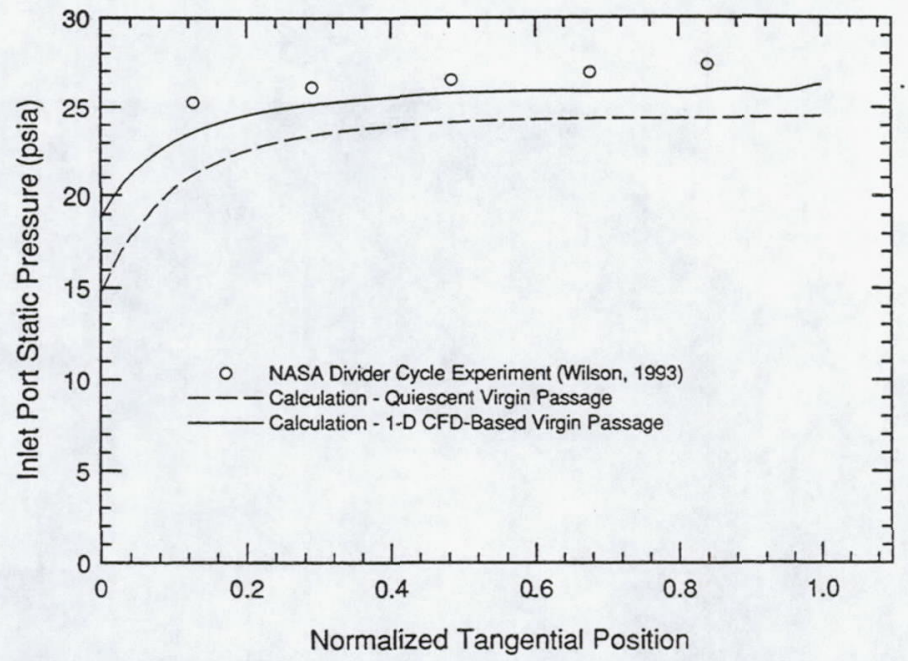

Figure 10. Comparison of measured and computed static pressure circumferential distribution in the inlet port of the NASA LeRC divider cycle experiment.

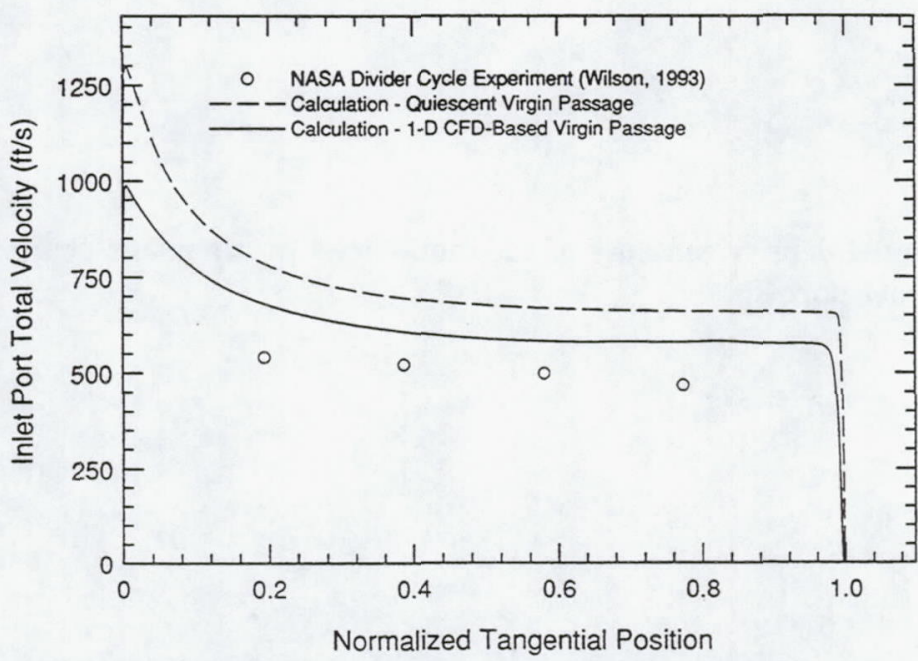

Figure 11. Comparison of measured and computed total velocity circumferential distribution in the inlet port of the NASA LeRC divider cycle experiment.

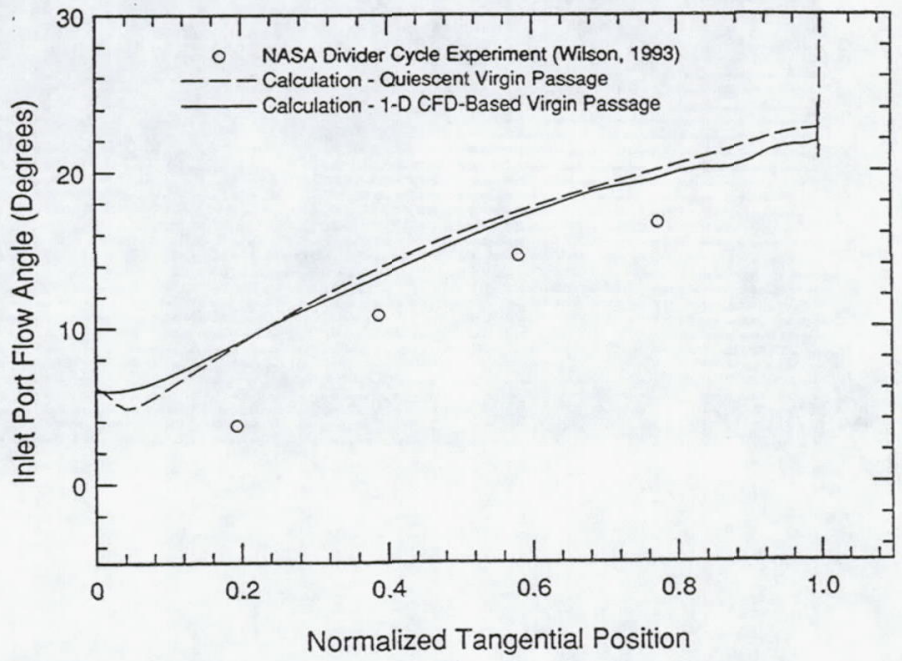

Figure 12. Comparison of measured and computed flow angle circumferential distribution in the inlet port of the NASA LeRC divider cycle experiment. 
Public reporting burden for this collection of information is estimated to average 1 hour per response, including the time for reviewing instructions, searching existing data sources, gathering and maintaining the data needed, and completing and reviewing the collection of information. Send comments regarding this burden estimate or any other aspect of this collection of information, including suggestions for reducing this burden, to Washington Headquarters Services, Directorate for Information Operations and Reports, 1215 Jefferson.

Davis Highway, Suite 1204, Arlington, VA 22202-4302, and to the Office of Management and Budget, Paperwork Reduction Project (0704-0188), Washington, DC 20503.

\begin{tabular}{|l|c|r|}
\hline 1. AGENCY USE ONLY (Leave blank) & $\begin{array}{r}\text { 2. REPORT DATE } \\
\text { February } 1994\end{array}$ & $\begin{array}{r}\text { 3. REPORT TYPE AND DATES COVERED } \\
\text { Technical Memorandum }\end{array}$ \\
\hline
\end{tabular}

\section{TITLE AND SUBTITLE}

5. FUNDING NUMBERS

Two-Dimensional CFD Modeling of Wave Rotor Flow Dynamics

6. AUTHOR(S)

WU-505-62-10

Gerard E. Welch and Rodrick V. Chima

\section{PERFORMING ORGANIZATION NAME(S) AND ADDRESS(ES)}

National Aeronautics and Space Administration

Lewis Research Center

Cleveland, Ohio 44135-3191

8. PERFORMING ORGANIZATION REPORT NUMBER

E-8404

\section{SPONSORING/MONITORING AGENCY NAME(S) AND ADDRESS(ES)}

10. SPONSORING/MONITORING AGENCY REPORT NUMBER

National Aeronautics and Space Administration

Washington, D.C. 20546-0001

NASA TM-106261

11. SUPPLEMENTARY NOTES

Prepared for the 11th Computational Fluid Dynamics Conference sponsored by the American Institute of Aeronautics and Astronautics, Orlando, Florida, July 6-9, 1993. Responsible person, Gerard E. Welch, organization code 2760, (216) 433-8003.

12a. DISTRIBUTION/AVAILABILITY STATEMENT 12b. DISTRIBUTION CODE

Unclassified - Unlimited

Subject Category 07

\section{ABSTRACT (Maximum 200 words)}

A two-dimensional Navier-Stokes solver developed for detailed study of wave rotor flow dynamics is described. The CFD model is helping characterize important loss mechanisms within the wave rotor. The wave rotor stationary ports and the moving rotor passages are resolved on multiple computational grid blocks. The finite-volume form of the thin-layer Navier-Stokes equations with laminar viscosity are integrated in time using a four-stage Runge-Kutta scheme. Roe's approximate Riemann solution scheme or the computationally less expensive Advection Upstream Splitting Method (AUSM) flux-splitting scheme is used to effect upwind-differencing of the inviscid flux terms, using cell interface primitive variables set by MUSCL-type interpolation. The diffusion terms are central-differenced. The solver is validated using a steady shock/laminar boundary layer interaction problem and an unsteady, inviscid wave rotor passage gradual opening problem. A model inlet port/passage charging problem is simulated and key features of the unsteady wave rotor flow field are identified. Lastly, the medium pressure inlet port and high pressure outlet port portion of the NASA Lewis Research Center experimental divider cycle is simulated and computed results are compared with experimental measurements. The model accurately predicts the wave timing within the rotor passages and the distribution of flow variables in the stationary inlet port region.

14. SUBJECT TERMS

CFD; Navier-Stokes equations; Wave rotor; Unsteady; Upwind schemes

15. NUMBER OF PAGES

16

16. PRICE CODE

$\mathrm{A} 03$

\begin{tabular}{|c|c|c|c|}
\hline $\begin{array}{c}\text { 17. SECURITY CLASSIFICATION } \\
\text { OF REPORT } \\
\text { Unclassified }\end{array}$ & $\begin{array}{c}\text { 18. SECURITY CLASSIFICATION } \\
\text { OF THIS PAGE } \\
\text { Unclassified }\end{array}$ & $\begin{array}{c}\text { 19. SECURITY CLASSIFICATION } \\
\text { OF ABSTRACT } \\
\text { Unclassified }\end{array}$ \\
\hline
\end{tabular}

